\title{
Rechtsgeschichte
}

http://www.rg-rechtsgeschichte.de/rg11

Zitiervorschlag: Rechtsgeschichte Rg 11 (2007)

$\operatorname{Rg} 112007$

$219-221$

http://dx.doi.org/10.12946/rg11/219-221

\section{Dietfrid Krause-Vilmar}

Keine Gnade 
Hoffnungen eingestehen musste; eine Bevölkerung, die gleichzeitig vom Staat abhängig und mit ihm unzufrieden ist, und ein Reservoir an Gegenhoffnungen auf die Bundesrepublik, die kaum realistischer waren als die Utopien der ersten Volksrichter über die Zukunft des Sozialismus.

Markovits hat mit ihrem spannend geschriebenen, so tatsachen- wie gedankenreichen Werk eine in der bisherigen Geschichte des Rechts vielleicht einmalige Gelegenheit beim Schopfe gepackt. Juristisch (nicht politisch) erklärt, ist sie eine Abwicklungsgewinnerin im Ergebnis des »Vertrages zwischen der Bundesrepublik Deutschland und der Deutschen Demokratischen Republik über die Herstellung der Einheit Deutschlands - Einigungsvertrag - « vom 3 I. August I990. Zum Nutzen aller Denkenden, der Rechten wie der Linken.

Hermann Klenner

\section{Keine Gnade*}

Im ersten Teil (Die Strafverfolgung von NSVerbrechen in der SBZ/DDR I945-I966) gibt Christian Dirks auf der Grundlage der neuesten Forschungen (z. B. von K. W. Fricke, A. Weinke, H. Wentker, F. Werkentin u. a.) einen zusammenfassenden Überblick über die sowjetischen Militärtribunale, deren flächendeckende Urteils- und Strafpraxis vor allem ab I 947 NS-Verbrecher in gleicher Weise wie Gegner und Oppositionelle des Regimes in der SBZ/DDR erfasste. Mit dem Befehl Nr. 20I der sowjetischen Militäradministration (August 1947) gingen die meisten Strafverfahren dann an ostdeutsche Gerichte über. Diese Verfahren widersprachen in der Regel eklatant rechtsstaatlichen Grundsätzen, zumal da nun die vielfach absolut grundlose Unterstellung »NS- und Kriegsverbrecher « mit der Enteignung von Oppositionellen oder auch, wie gerade an einem Fallbeispiel gezeigt wurde, von einem bereits in der Nazizeit "arisierten « deutsch-jüdischen Industriellen amalgamiert wurde. ${ }^{\mathbf{I}}$

Dirks berichtet auch über die Waldheimer Prozesse, in denen besonders eingerichtete Straf- kammern des Landgerichts Chemnitz im Frühjahr I950 3385 bislang Internierte in Schnellverfahren aburteilten, wobei der Anteil der tatsächlich an NS-Verbrechen Beteiligten kaum rekonstruierbar ist. Im Anschluss an die Waldheimer Prozesse nahm die Verfolgung der NSVerbrechen in der DDR spürbar ab. Zu den Ergebnissen dieser Arbeit gehört die Erkenntnis, dass es eine systematische Verfolgung der NSVerbrechen in der DDR in den 5oer Jahren ebenso wenig gab wie in der Bundesrepublik. Die dann in der DDR einsetzende Kampagnenpolitik mit den Schauprozessen gegen Theodor Oberländer, Hans Globke u. a. erweist sich als instrumentell, nicht als selbstkritisch-reflexiv. Schuld waren eben »die anderen «. So zutreffend in fast allen "Fällen « die historischen Beweise gegen Globke, Oberländer und die anderen waren, so fragwürdig und durchsichtig war das in unerträglich selbstherrlicher Tonlage vorgetragene instrumentelle Motiv der vermeintlichen "Enttarnung « des »faschistischen « Charakters der »Bonner Politik«.

\footnotetext{
* Christian Dirks, "Die Verbrechen der anderen «. Auschwitz und der Auschwitz-Prozess der DDR: Das Verfahren gegen den KZ-Arzt Dr. Horst Fischer, Paderborn: Ferdinand Schöningh 2006, 406 S., ISBN 3-506-7I363-9
}

I Götz Aly, Michael SontheiMER, Fromms. Wie der jüdische Kondomfabrikant Julius F. unter die deutschen Räuber fiel, Frankfurt am Main 2007. 
Der zweite Teil ist der Biographie Horst Fischers gewidmet. Er beruht auf eigenen Forschungen des Verfassers und der Auswertung bislang nicht veröffentlichter und wissenschaftlich nicht bekannter Quellen. Vorangestellt ist diesem Teil eine ausgezeichnete Darstellung der Rolle der SS-Ärzte in Auschwitz. Insbesondere die maßgebliche "Aufgabe « des SS-Standortarztes und seines Stellvertreters (dies war lange Jahre Fischer) ist m.E. in dieser Genauigkeit noch nicht dokumentiert worden. Die Macht der SS-Lagerärzte, die als Akademiker bereits Fachautorität beanspruchen konnten, resultierte aus der von ihnen verantworteten und durchgeführten Selektion an der Rampe und in den Häftlings-Krankenbauten, auch in den Nebenlagern wie z. B. in Monowitz. Die persönlichen Niederschriften und die Vernehmungsprotokolle Fischers stellen »die mit Abstand umfangreichsten und detailliertesten Angaben zu den Tätigkeitsbereichen der Mediziner in den nationalsozialistischen Konzentrations- und Vernichtungslagern dar, die je ein SS-Arzt abgegeben hat (2 IO)

Was einen Menschen wie Horst Fischer dahin brachte, kontinuierlich diese Massenmorde zu veranlassen und sich an ihnen zu beteiligen, wird trotz der äußerst sorgfältigen und quellengestützten Rekonstruktion der Tätigkeit Fischers im letzten nicht deutlich, die Frage ist wahrscheinlich auch nicht schlüssig zu beantworten. In seinem Umfeld tätig gewesene ehemalige Häftlinge entlasteten Fischer bei späteren Aussagen teilweise, indem sie auf Zurückstellungen Todgeweihter, auf Hilfeleistungen und Ambivalenzen hinwiesen. Überliefert ist ein Versuch Fischers, sich angesichts des Grauens versetzen zu lassen; dieser blieb erfolglos. Die überwiegend späteren Äußerungen Fischers hierzu, die Dirks mitteilt, wirken hilflos und ehrlich zugleich. Sie beschönigen und rechtfertigen nichts, und doch wird die Person nicht ganz erkennbar. So bleiben die bekannten Erklärungsversuche wie Karrierestreben, Antisemitismus, Bereicherung, Verstrickung übrig, für die wir jedoch bei Fischer keinen schlagenden Beweis finden. Das einzige, was sich mir bei der Lektüre aufdrängte, war der Eindruck eines Menschen, der gegebenen Verhältnissen und Herausforderungen nichts oder nur wenig entgegensetzen konnte oder wollte.

Der dritte umfangreichste Teil - wie der zweite ausschließlich auf eigenen Forschungen des Verfassers basierend und ebenfalls neue, bislang unbekannte Quellen auswertend - ist dem Prozess gegen Fischer vor dem Obersten Gericht der DDR im Jahre 1966 gewidmet. Dirks betritt hier - ebenso wie im zweiten Teil - Neuland: Erstmals wird dieses - als Reaktion auf die Frankfurter Auschwitz-Prozesse - geführte Gerichtsverfahren auf der Grundlage neuer Quellen, insbesondere der umfangreichen Unterlagen des ehemaligen Ministeriums für Staatssicherheit (MfS), in allen Einzelheiten akribisch rekonstruiert und unter zeitgeschichtlichen Aspekten exzellent ausgeleuchtet. Die Darstellung des Verfahrens setzt durchaus Maßstäbe, vor allem für die politische und gesellschaftliche Kontextualisierung rechtsgeschichtlicher Ereignisse. Das Verfahren entsprach in kaum einem Punkt rechtsstaatlichen Prinzipien, vielmehr wies es alle für einen Schauprozess einschlägigen Merkmale auf, wie Dirks plausibel macht. Auf Grund von MfS-Akten, die der Wissenschaft bislang noch nicht bekannt waren, kann er nachweisen, dass das Verfahren von Anbeginn an bis zum bitteren Ende - Fischer wurde mit dem Tode bestraft und guillotiniert - vom Geheimdienst der DDR gesteuert und in enger Absprache mit dem Politbüro der SED, d.h. mit der politischen Führung der DDR, durchgeführt wurde. Dirks weist aus 
den Akten nach, dass das Verfahren vom Geheimdienst mit dem Ziel geführt werden sollte, die Politik der SED »hinsichtlich der Entlarvung der Revanchepolitik und der Rehabilitierungsbestrebungen des Bonner Staates « zu unterstützen. Insbesondere sollte das Zusammenwirken der IG Farben mit der SS ermittelt werden (209).

Nur durch einen Zufall war man im Jahre I965 auf den in Spreenhagen (Brandenburg) praktizierenden Landarzt Dr. Horst Fischer gestoßen und hätte seine historische Rolle vermutlich nicht entdeckt, wenn man nicht wegen seiner häufigen Westkontakte und seiner "politischen Unzuverlässigkeit « einen »Informator « auf ihn angesetzt hätte. Es gab eben - der eigenen Legendenbildung zum Trotz - in der SBZ/DDR keine systematische Verfolgung der NS-Verbrechen und NS-Täter. Dirks weist darauf hin, dass die pharisäerhafte Vorwurfshaltung der DDRFührung gegenüber der BRD (Braunbuch, Oberländer, Globke u.a.), da die Dokumente im Einzelnen meist zutrafen, gegen die eigene Intention im Westen durchaus die kritische Bearbeitung des Nationalsozialismus stärkten. Im Osten täuschten dagegen einzelne ausgewählte Schauprozesse wie in Waldheim und eben gegen Dr. Fischer eine konsequente Bearbeitung der NS-Zeit vor. - Zu den zahlreichen Ergebnissen dieser verdienstvollen Untersuchung gehört auch der Hinweis darauf, dass Fischers Aussagen in
Ost-Berlin hilfreich für den zweiten Frankfurter Auschwitz-Prozess (335) waren.

Dr. Horst Fischer hatte vor Gericht unumwunden - und ohne sich als Opfer höherer Befehle hinzustellen - seine Verbrechen eingestanden und in einzigartiger Weise an der Aufklärung der Tatumstände mitgewirkt. Kein KZArzt hat in derart sich selbst nicht schonender Weise einem Gericht gedient. Dies gewinnt noch deutlichere Konturen, wenn man an die Verteidigungsstrategien der im Frankfurter AuschwitzProzess Angeklagten denkt. Auffallend und bedrückend ist für mich, dass es für ihn, der im "Neuen Deutschland « als "Bestie « diffamiert wurde, weder mildernde Umstände noch einen Gnadenerweis gab. Entlastende Zeugen wie Hermann Langbein und Karl Lill wurden bewusst nicht geladen, ihre schriftlichen Einlassungen wurden nicht näher berücksichtigt. Die Argumente seines Verteidigers Dr. Vogel wurden bei der Urteilsbildung nicht berücksichtigt. Fischers Schuldeingeständnis und seine ehrlich bezeugte Ambivalenz, seine gelegentliche Hilfsbereitschaft und sein Zögern bei einigen Selektionen wurden übergangen. Schließlich wurde das Gnadengesuch der Verteidigung, die für lebenslange Haft plädiert hatte, von Erich Mielke und Walter Ulbricht abgelehnt.

\section{Dietfrid Krause-Vilmar}

